REFERENCIA: Echauri Galván, B. (2020). TraducciONG: potencialidades del aprendizaje-servicio en asignaturas de traducción a través de una experiencia didáctica. ENSAYOS, Revista de la Facultad de Educación de Albacete, 35(2). Enlace web: http://www.revista.uclm.es/index.php/ensayos - Consultada en fecha (dd-mm-aaaa)

\title{
TRADUCCIONG: POTENCIALIDADES DEL APRENDIZAJE-SERVICIO EN ASIGNATURAS DE TRADUCCIÓN A TRAVÉS DE UNA EXPERIENCIA DIDÁCTICA
}

\section{TRANSLATINGO: THE POTENTIALITIES OF SERVICE LEARNING FOR TRANSLATION STUDIES THROUGH A TEACHING EXPERIENCE}

\author{
Bruno Echauri Galván \\ bruno.echauri@uah.es \\ Facultad de Filosofía y Letras. Universidad de Alcalá (España)
}

Recibido: $11 / 06 / 2020$

Aceptado: 09/12/2020

\begin{abstract}
Resumen:
El presente artículo describe una experiencia educativa que incorpora el aprendizaje-servicio a los estudios de grado en traducción. Para ello, los estudiantes del tercer curso del grado en Lenguas Modernas y Traducción de la Universidad de Alcalá han colaborado con la ONG Manos Unidas en la traducción del español al inglés de material educativo centrado en los derechos de los niños y el desarrollo sostenible. Al margen de estudiar y presentar los beneficios de esta iniciativa concreta, este artículo busca también realzar la idoneidad de los estudios en traducción como campo para el desarrollo de proyectos de aprendizaje-servicio. Para explorar ambos puntos, se ha recabado la opinión de los distintos participantes (estudiantes y miembros de la ONG) a través de dos encuestas que confirman el impacto positivo de esta metodología y animan a promover la planificación e implementación de propuestas similares en años venideros.
\end{abstract}

Palabras clave: educación superior; aprendizaje-servicio; traducción; ONG

\begin{abstract}
:
The present paper describes a teaching experience that implements service learning in translation studies at an undergraduate level. In this regard, students in the third year of the BA in Modern Languages and Translation at the University of Alcalá have collaborated with the NGO Manos Unidas in the English translation of educational material on children's rights and sustainable development. In addition to studying and presenting the potential benefits of this particular proposal, this paper also seeks to foreground the suitability of translation as a field in which service learning projects can be developed. In order to explore the aforementioned points, two surveys (one addressed at students and the other at the members of the NGO) were conducted. Results confirm the positive impact of this methodology and encourage further planning and implementation of similar initiatives in upcoming years.
\end{abstract}

Keywords: higher education; service learning; translation; NGO 


\section{Introducción}

El presente artículo describe una experiencia educativa realizada en la Universidad de Alcalá dentro de la asignatura Fundamentos de la Traducción. En ella participaron 26 estudiantes del tercer curso del grado en Lenguas Modernas y Traducción que trabajaron en grupos a lo largo de todo un cuatrimestre. Esta acción docente se plantea como una experiencia de aprendizajeservicio (ApS) en colaboración con Manos Unidas Guadalajara y se estructura en torno a la traducción al inglés de los distintos materiales que conforman una exposición interactiva que la ONG organiza en centros educativos de la región. A través de esta tarea, se busca consolidar contenidos teóricos y destrezas prácticas de la asignatura y el grado, al tiempo que se potencian aspectos formativos derivados del ApS como la educación en valores. En consonancia con lo anterior, los objetivos generales del proyecto son los siguientes:

- Reivindicar las asignaturas de traducción como plataformas para fortalecer la implementación del ApS en la Universidad.

- Diseñar e implementar una experiencia de ApS que conexione la teoría y la práctica en una asignatura de traducción a nivel de grado.

- Validar esta metodología como herramienta didáctica adecuada para los estudios de traducción a partir de la respuesta de los colectivos involucrados en el proyecto.

\section{Fundamentación teórica}

\subsection{Aprendizaje-servicio en la Universidad}

El aprendizaje-servicio puede definirse someramente como una metodología pedagógica en la que el proceso de enseñanza-aprendizaje dispuesto en el currículo académico se orienta a la aplicación de conocimientos de tal forma que estos cubran necesidades sociales de la comunidad. Este componente social de la enseñanza aparece recogido en el Marco común europeo de referencia para las lenguas (Council of Europe, 2018), donde la responsabilidad y la cohesión social, así como el papel del aprendiente para lograrlas, se subrayan como objetivos seminales básicos y donde se promueven varias competencias generales a tal efecto.

Esta mezcla entre pedagogía y participación social sobre la que se fundamentan los proyectos de ApS puede reportar numerosos beneficios a todas las partes implicadas y en distintos planos. Para Rodríguez-Gallego (2014), estos beneficios inciden, fundamentalmente, sobre tres ejes:

- El currículum académico, puesto que el ApS impulsa la formación práctica y la adquisición de unos contenidos teóricos mejor adaptados al contexto social, económico y profesional del aprendiente.

- La educación en valores como la prosocialidad, la solidaridad o la responsabilidad social que, a su vez, ayudan a formar profesionales más cívicos y concienciados.

- La vinculación con la comunidad, ya que se interviene sobre problemáticas reales que surgen de necesidades concretas de la sociedad circundante.

Otros listados, como el propuesto por Martín y Puig (2018), amplían la relación anterior y añaden nuevas dimensiones que incluyen la realización personal y la mejora en la autoestima a partir de una acción que reporta un beneficio a los demás y ayuda al aprendiente a sentirse útil, o el incremento del interés del alumnado por lo que ocurre a su alrededor y por participar en iniciativas que promuevan la mejora de ese entorno. 
Sin embargo, la implementación de proyectos de este tipo en el ámbito universitario no es simplemente provechosa, sino también y sobre todo, necesaria. Como apunta Martínez (2018), la Universidad es una pieza fundamental del engranaje formativo, una institución por la que pasarán muchas de las mentes que construyan y sostengan el mañana. Por consiguiente, no puede ni debe aspirar a ser una simple fábrica de buenos profesionales, sino que debe potenciar también el factor humano para contribuir a fortalecer la sociedad que la rodea $y$, de este modo, fortalecerse a sí misma. En una línea similar, Martínez subraya que el ApS no es simplemente una forma de conectar el aprendizaje del alumnado con la sociedad para crear una relación simbiótica que beneficie a ambas partes; es también un modo de que la Universidad eche verdaderas raíces en su entorno más inmediato, del que se nutre y al que debe nutrir.

Pese a ser un movimiento originado fundamentalmente en el mundo anglosajón y en América Latina (Rodríguez-Gallego, 2014), donde cuenta con un largo recorrido y aceptación, el aprendizaje-servicio como marco metodológico en la enseñanza universitaria española ha cristalizado ya en numerosas aplicaciones que atraviesan diferentes contextos y disciplinas. Como botón de muestra, Rodríguez-Gallego (2014) centra su propuesta en el ámbito de la Didáctica General mientras que Pérez-Pueyo et al. (2019) lo hacen en el de la Educación Física. Otros autores como Madrid (2008) llevan el ApS al Derecho e iniciativas como la de FranciscoAmat y Moliner-Miravet (2010) describen acciones docentes más transversales que implican a distintas disciplinas como la Educación y la Comunicación Audiovisual. Del mismo modo, varias universidades de nuestro país como la Universidad Politécnica de Valencia (http://www.upv.es/entidades/CCD/infoweb/ccd/info/954671normalc.html), la Universidad de Alcalá (https://www3.uah.es/ice/observatorio-aps.html), la Universidad de Valencia (https://www.uv.es/uvweb/centro-formacion-calidad-manuel-sanchis-guarner/es/capsa/apsuv/capsa-1286022919165.html) o la Universidad Complutense de Madrid (https://www.ucm.es/diversidad/aprendizaje-servicio), entre otras, han impulsado la constitución de unidades y observatorios orientados a la promoción, creación y difusión de iniciativas fundamentadas en esta metodología de aprendizaje.

El párrafo anterior prueba la heterogeneidad de las propuestas relacionadas con el aprendizajeservicio y lo holístico de una metodología que puede adaptarse a muy diversos entornos educativos. Sin embargo, con contadas excepciones como los proyectos de Tamayo (2016), centrado en la subtitulación para personas sordas, o el de Gil-Sanromán (2019), que lleva el ApS al aula de traducción jurídica inglés-español, escasean a día de hoy en nuestro país las experiencias docentes que unan este marco metodológico con la traducción de manera explícita. Esto resulta cuando menos sorprendente, en tanto en cuanto vivimos en una realidad multicultural y plurilingüística en la que la relación entre ambos podría resultar extremadamente fructífera y beneficiosa. De hecho, esta conexión sí se ha explorado con éxito y mayor profundidad en universidades de otros países como Estados Unidos (Bugel, 2013; FaszerMcMahon, 2013). La experiencia didáctica que se describe en este artículo busca continuar llenando este vacío y presentar las asignaturas y grados en traducción e interpretación como un contexto idóneo en el que llevar a cabo proyectos educativos que ayuden a cubrir necesidades sociales en nuestros barrios y nuestras ciudades.

\subsection{La traducción y las ONG en España}

Pocos entornos son más propicios para el empleo de la traducción como herramienta de igualdad social que los servicios públicos. Una larga lista de trabajos como los de Sales (2005), Vázquez-Navarrete, et al. (2009) o Corsellis et al. (2010) demuestran la necesidad de la traducción e interpretación en estas instituciones al tiempo que nos enfrentan con un panorama crítico en el que las demandas muchas veces no son escuchadas y en el que la escasez de 
recursos es un problema recurrente. Estas lagunas (y tantas otras) han tenido que ser cubiertas con sumo esfuerzo por organizaciones sociales sin ánimo de lucro como las ONG.

Tal y como señala Revilla-Blanco (2015), las ONG surgieron como fenómeno en nuestro país a finales de los 80. Lejos de ser una moda pasajera, el paso de los años y los distintos contextos socioeconómicos que en estas décadas ha atravesado España no han hecho sino consolidar el peso de estas instituciones en nuestra sociedad. Si bien la crisis de 2008 puso en peligro gran parte de su financiación, también sirvió para que las ONG pasaran a formar parte integrante del tejido social español presente y futuro (Revilla-Blanco, 2015).

La actividad de estas organizaciones es, como se sabe, muy variada, y abarca ámbitos de actuación muy diferentes: nacionales e internacionales, asistenciales, sanitarios, educativos...todos ellos con numerosas ramificaciones adicionales. A las circunstancias propias de cada individuo o colectivo al que se pretende apoyar en estas esferas se suma, en muchos casos, una problemática añadida: las barreras lingüísticas y culturales. Es por ello que disciplinas como la traducción y la interpretación juegan un papel central en una red que busca prestar apoyo a la población inmigrante en la mayor cantidad de frentes posibles.

Como destacan Valero-Garcés y Cata (2006), la traducción en este tipo de organizaciones se aplica a muy distintos objetivos y se realiza en colaboración con entidades e instituciones de lo más variado, como centros de acogida, comisarías, juzgados, hospitales y otros centros de salud o servicios sociales, por citar solo algunos ejemplos. Esta heterogeneidad entraña, lógicamente, lidiar con un abanico de documentos de trabajo de lo más diverso. Desde solicitudes de asilo y la cantidad ingente de documentación que de ellas se deriva hasta textos de carácter sanitario, pasando por los trámites administrativos inherentes a la escolarización o la inscripción en distintas instituciones o registros, los traductores que colaboran con una ONG, normalmente voluntarios, deben afrontar múltiples desafíos enmarcados en situaciones vitales complejas. Lo delicado de los materiales con los que trabajan pone de relieve su rol decisivo en las acciones que llevan a cabo las ONG españolas para un normal asentamiento, integración y desarrollo de la población inmigrante en nuestro país.

Sin embargo, las organizaciones sin ánimo de lucro no son solo redes de seguridad para momentos cruciales o de crisis ni su labor meramente reactiva; también destinan gran parte de sus esfuerzos a la concienciación, la formación y la educación en valores de distintas capas de la población. Al margen de servicios como los mencionados en el párrafo anterior, estas entidades ofrecen talleres formativos en materias diversas, organizan concursos, distribuyen material pedagógico, articulan campañas informativas a través de internet y otros medios...Estos y otros tantos proyectos tienen como objetivo un público heterogéneo que comprende amplios rangos de edad y procedencia. La traducción puede aquí, de nuevo, convertirse en una herramienta útil para llegar a parte de este público o multiplicar las posibilidades de difusión y posibilitar que distintas iniciativas alcancen foros y grupos impensables con propuestas de carácter monolingüe. La acción docente de ApS que se detalla en las siguientes páginas es una muestra de ello.

\section{Metodología}

La presente experiencia de ApS persigue los tres objetivos planteados en la introducción y se construye sobre las premisas propuestas por Martínez-Odría (2005), quien lista cinco requisitos fundamentales que las iniciativas de este tipo deben cumplir: realización de un proyecto de servicio que sea presentado a la comunidad; respuesta a una necesidad real de dicha comunidad; protagonismo del alumnado en el proceso de aprendizaje y participación social; conexión de la actividad con los contenidos y objetivos curriculares; reflexión constante y búsqueda de nexos con lo aprendido en las aulas. Como podrá comprobarse a lo largo de las 
siguientes secciones, esta es una iniciativa planificada de la que el estudiante es siempre parte central y que moviliza conocimientos adquiridos en el desarrollo de la asignatura y de sus estudios universitarios. Asimismo, invita al alumnado a la reflexión y evaluación crítica a través de varios talleres incluidos en las distintas fases del proyecto. Por último, la colaboración con una ONG como Manos Unidas Guadalajara asegura su papel como respuesta a una carencia social y la ulterior presentación y utilización del resultado fuera de las aulas.

Tomando el ApS como marco metodológico general, esta propuesta se concreta a través de un trabajo colaborativo que, como se describe en la sección 3.2.2, sigue dinámicas diferentes en función de la etapa del proyecto en la que se encuentren los aprendientes. Este modelo de trabajo establece el diálogo y la comunicación constante entre las partes como motores de un esfuerzo equilibrado, recíproco y compartido a partir del cual alcanzar los objetivos de un proyecto (Maldonado-Pérez, 2007). Bien ejecutado, este sistema facilita el aprendizaje de calidad de los estudiantes, al tiempo que los hace responsables de la formación de otros compañeros y ayuda a limar las asimetrías de conocimientos entre pares (Maldonado-Pérez y Sánchez, 2012). Estos dos últimos puntos son especialmente relevantes en asignaturas de traducción, orientadas, fundamentalmente, al desarrollo de lo que se conoce como "competencia traductora».

Kelly (2002) definió este concepto ${ }^{1}$ como una macrocompetencia que engloba todo el conjunto de destrezas, conocimientos, actitudes y capacidades propios de la traducción como actividad experta y que un traductor profesional debe reunir. Dentro de esta macrocompetencia, autores como Mareschal (2005) destacan al menos seis microcompetencias, a saber: traductológica, lingüística, temática, informática, de redacción y de investigación a las que podrían sumarse otras como, por ejemplo, la intercultural. En vista de todo lo anterior, puede colegirse que dominar la competencia traductológica dista de ser algo sencillo. Y es que traducir, huelga decirlo, no es tarea fácil. Hay que tener en cuenta numerosas variables y conjugar un amplio abanico de conocimientos a lo largo de un proceso laborioso y complejo. En este escenario, es lógico suponer que la adquisición de la competencia traductora avanza a distintas velocidades entre los alumnos, no solo en términos generales, sino también en lo que al desarrollo de cada microcompetencia se refiere. Así las cosas, el trabajo colaborativo se presenta como una herramienta potencialmente eficaz a través de la cual los estudiantes pueden compartir sus fortalezas y compensar sus debilidades a partir de un apoyo mutuo que ayude a acompasar los ritmos de aprendizaje y adquisición de conocimientos.

\subsection{Contexto de aplicación}

Esta acción docente se enmarca dentro de la asignatura Fundamentos de la Traducción, impartida con carácter obligatorio en el segundo cuatrimestre del tercer curso del grado en Lenguas Modernas y Traducción de la Universidad de Alcalá. En el programa de la asignatura se abordan distintas corrientes teóricas dentro de la historia de la traducción, desde el funcionalismo hasta el giro cultural, pasando por enfoques filosóficos o por conceptos tan importantes como la (in)visibilidad del traductor. A los contenidos teóricos impartidos les acompaña una importante vertiente práctica en la que los estudiantes deben trabajar con los distintos conceptos explicados en las sesiones magistrales, así como aplicar algunos de los enfoques abordados a lo largo del curso. En relación con este último punto, las actividades desarrolladas durante el cuatrimestre son muy variadas, abarcando traducciones directas e inversas, textos meta (TM) orientados a distintas audiencias, trabajos de domesticación o extranjerización textual o reescrituras, entre otras.

\footnotetext{
${ }^{1}$ Para conocer otras definiciones del concepto, pueden consultarse los trabajos de Bell (1991), HurtadoAlbir (1996) o Neubert (2000), entre otros.
} 
El proyecto que aquí se presenta permite que los 26 discentes involucrados apliquen varios de los conocimientos listados anteriormente a una experiencia de calado social, con un carácter más profesional y con el potencial para trascender el aula y la audiencia cautiva a la que normalmente dirigen sus trabajos. Así, la tarea les ayuda a consolidar parte del temario y desarrollar varias de las competencias específicas de la asignatura, como ser capaces de traducir diferentes tipos de textos, aplicar modelos teóricos concretos al proceso traductológico, tomar decisiones adecuadas en función de la audiencia de destino o analizar de forma crítica su propia práctica de la traducción; pero también se concibe como una iniciativa holística y transversal en la que se emplean conocimientos adquiridos en otras materias, como el manejo de fuentes terminológicas o el uso de algunas herramientas informáticas durante el proceso de traducción o en el tratamiento de las imágenes.

Por otro lado, en línea con el abanico tripartito de beneficios que describía Rodríguez-Gallego (2014), esta propuesta busca que la práctica docente no descuide la educación en valores y la conexión del estudiantado con su comunidad y entorno. Su colaboración con una ONG cercana como Manos Unidas Guadalajara y el trabajo con un texto que toca preocupaciones nucleares de hoy en día como los derechos de los niños y el desarrollo sostenible crean un ecosistema idóneo para tratar de alcanzar una mayor responsabilidad cívica colectiva intentando construir unos principios morales más sólidos y una implicación más firme y sostenida. Asimismo, la naturaleza del encargo y su audiencia de destino (véase sección 3.2.1) no solo coadyuvan a concienciar al estudiantado sobre los problemas que les y nos rodean, sino también y quizás más significativo, de la importancia de educar sobre ellos.

Por último, como se describirá con detalle más adelante, la tarea se vehicula a través de un trabajo en grupo organizado por fases entre las que se intercalan talleres de reflexión y que culmina con una presentación oral ante los miembros de la ONG involucrados en el proyecto, lo que refuerza competencias genéricas como el trabajo colaborativo, el pensamiento crítico o la comunicación oral. De este modo, el ApS se convierte en una plataforma abarcadora en la que se entrelazan competencias específicas y transversales para cristalizar en una acción docente concreta con un impacto personal inmediato y una transcendencia social a medio plazo.

\subsection{Desarrollo}

\subsubsection{Texto original}

La presente experiencia educativa entronca con la estrategia de la ONG Manos Unidas de traducir gran parte de sus materiales a distintas lenguas para hacerlos llegar al mayor número posible de personas. Dentro de esos grupos meta se encuentra también la población infantil, para la que ya se han traducido, a varias lenguas, historias como las de Súper Pepo, un personaje recurrente en las iniciativas de la organización alrededor del cual se organizan tramas que dan a conocer problemas como el hambre, la contaminación o la pobreza, al tiempo que promueven distintos valores relacionados con los derechos de los niños o los objetivos del milenio, entre otros. De este modo, la ONG busca crear materiales fácilmente asimilables por los más pequeños para tratar de sensibilizarlos y formarlos de una forma divertida y amena.

La acción docente propuesta se enmarca dentro de esta serie sobre Súper Pepo, aunque el texto original con el que trabajaron los estudiantes no es un relato o historia como tal. En este caso, el proyecto se organiza en torno a una exposición interactiva protagonizada por este pequeño superhéroe ${ }^{2}$. Esta exposición se compone de paneles de gran tamaño que se disponen formando

\footnotetext{
2 Para más información sobre esta exposición y su funcionamiento pueden consultarse la propia web de la ONG (https://www.manosunidas.org/) o vídeos como el que se adjunta en el siguiente enlace: https://www.youtube.com/watch?v=oQIBXGooWSE
} 
un recorrido por el que los participantes deberán pasar superando diversas pruebas. En este camino les acompañará una persona de la organización que les irá presentando y ayudando en las distintas actividades, al tiempo que comparte información relacionada con los derechos de los niños, los Objetivos de Desarrollo Sostenible de las Naciones Unidas y algunos de los problemas que estos objetivos buscan paliar (el hambre, la contaminación, la falta de acceso a la sanidad y la educación), personificados en malvados supervillanos. El propósito final de la exposición es que los niños y niñas descubran que, al igual que Súper Pepo, ellos mismos pueden ser auténticos superhéroes con el poder para cambiar el mundo, un poder que no pasa por volar - lanzar rayos por los ojos, sino por acciones cotidianas con un impacto real e inmediato en nuestro entorno.

Como se avanzaba en el párrafo anterior, la exposición se organiza a partir de unos paneles donde se recogen las actividades a realizar, sus directrices, datos interesantes sobre los temas que se abordan durante el recorrido o pautas para acceder a contenidos interactivos a través de dispositivos digitales. Son precisamente estos paneles junto con las explicaciones que deben dar los guías de la exposición lo que los estudiantes de la Universidad de Alcalá recibieron como texto original (TO) y lo que tendrían que traducir al inglés a lo largo de las distintas fases del proyecto para dar forma a un único TM final. La intención de la ONG es utilizar la guía y los paneles traducidos para poder desarrollar la exposición en lengua inglesa en distintos centros educativos de la provincia de Guadalajara y alrededores. Así, la compleción de esta propuesta de ApS permitirá que mensajes tan importantes como los que traslada esta iniciativa de Manos Unidas gocen de una mayor difusión: la posibilidad de organizar la actividad en dos lenguas amplía el número de potenciales destinatarios, multiplica su atractivo y, además, la sitúa en consonancia con el auge de los centros bilingües y el crecimiento exponencial del peso del inglés en nuestro sistema educativo en los últimos años.

\subsubsection{Secuenciación del trabajo}

Tal y como se anticipaba en la sección previa, la meta final de los estudiantes era conseguir una única traducción inversa al inglés de los paneles para la exposición de Súper Pepo y las explicaciones que la acompañan. Para completar este encargo, su labor se organiza en cuatro fases construidas sobre tres puntos axiales. El primero es, por supuesto, el trabajo fuera del aula que los equipos deben ir completando en los plazos indicados. El segundo es una serie de talleres distribuidos a lo largo del cuatrimestre que marcan el ritmo de la tarea. Estas sesiones cumplen una doble función. Por un lado, ofrecen un espacio para la reflexión, el comentario y el análisis de los desafíos traductológicos del texto y cómo estos conexionan con los contenidos del curso, del grado y de otras actividades completadas a lo largo de la asignatura. Por otro, sirven también como foro de discusión sobre la vertiente cívica y social de la tarea. En este sentido, los talleres operan como herramienta con la que pulsar las impresiones iniciales del estudiantado con respecto a aspectos nucleares de este proyecto. Así, en cada uno de ellos se lanzan varias preguntas relacionadas con las cuestiones recogidas en la encuesta que tendrán que completar ulteriormente (véase sección 3.3). Se estima que esta práctica puede resultar útil como fuente de datos adicional, como forma de recoger el sentir general del grupo y como mecanismo para calibrar su reacción espontánea ante ciertos interrogantes. El tercer y último eje estructural son las tutorías de apoyo que cada grupo o estudiante puede solicitar en el momento que lo desee.

Antes de entrar en mayor detalle, conviene subrayar que la organización inicial de la acción docente se vio forzosa y ligeramente alterada por la crisis del coronavirus. Toda actividad presencial pasó a realizarse en línea a partir del mes de marzo, utilizando para ello la plataforma Blackboard y sus distintas herramientas. Del mismo modo, algunas acciones previstas dentro del proyecto, como las visitas de los miembros de la ONG a la Universidad de Alcalá o de los estudiantes a los centros donde se estaba realizando la exposición en español, tuvieron que suspenderse. 
El pistoletazo de salida a la fase uno del proyecto se da en las primeras semanas del curso. En un taller introductorio, se les presenta el TO con el que van a trabajar, se explica de qué trata la exposición interactiva de Súper Pepo y se detallan otros aspectos como el público al que irá dirigida cada parte del TO y el uso que se pretende dar a su TM una vez completo y publicado. A continuación, se desgranan las pautas de trabajo para esta primera etapa de la tarea. En este estadio, se divide a los discentes en grupos de entre tres y cinco personas con el objetivo de traducir el texto original y obtener 6 versiones diferentes.

Para completar este encargo inicial disponen de un mes y medio. Pasadas tres semanas, se programa el primer taller de control y reflexión en el que, en un primer momento, se plantean los principales problemas que los grupos están teniendo a la hora de llevar a cabo sus traducciones. Puesto que no se trata de un texto excesivamente complicado, muchas de estas dificultades no orbitan en torno al ámbito terminológico o la complejidad de las estructuras gramaticales, sino que se centran en cómo obtener la naturalidad necesaria en un TM traducido a una lengua extranjera, en la adecuación del léxico a la edad de la audiencia meta y a la intención pedagógica del producto y en aspectos más específicos como las decisiones relativas a los nombres propios o al lenguaje inclusivo que permea la versión en español. Tres grupos plantean también problemas referentes al tratamiento de imágenes y el texto incluido en ellas. Con todo esto sobre la mesa, se abre un tiempo de puesta en común y de sugerencia de alternativas y soluciones supervisado por el profesor. Posteriormente, comienza una segunda parte del taller dedicada a reflexionar sobre los objetivos del proyecto, su trasfondo social, la relevancia de los temas que aborda el documento con el que están trabajando y el impacto que todo esto tiene en los estudiantes a nivel personal y motivacional. A este respecto, cabe recalcar el consenso en torno a la importancia de la temática de la exposición, la necesidad de formar a niños y niñas en los que son sus derechos y deberes como ciudadanos y la motivación que genera entre el alumnado sentirse una pieza más del engranaje y funcionar como el altavoz que permite difundir e inculcar estas ideas y valores a una audiencia aún mayor.

Tres semanas después del primer taller, los estudiantes hacen entrega de sus trabajos al profesor, quien lleva a cabo una primera corrección y se los devuelve pasados diez días. En este momento, se dan las directrices correspondientes a la segunda fase del proyecto. En primer lugar, cada grupo deberá perfeccionar sus traducciones a partir de los comentarios y correcciones del profesor. Posteriormente, todo grupo se unirá a otro para formar un total de tres equipos. Cada equipo deberá dar forma a un TM común, lo que implica que sus integrantes deberán centrarse en conjugar lo mejor de cada traducción anterior, llegar a puntos de acuerdo en aspectos dilemáticos y hacer una revisión exhaustiva del texto para entregar una versión lo más pulida posible.

Esta entrega se realiza coincidiendo con el segundo taller que, en este caso y dadas las circunstancias precitadas, ya se lleva a cabo de forma virtual. Al margen de retomar cuestiones abordadas en la reunión anterior (problemas en la traducción, dudas sobre el TO o el TM, entre otras) se vuelve a invitar a los discentes a reflexionar sobre otros aspectos relacionados con el proyecto. En concreto, se busca que compartan sus experiencias trabajando en grupo, su opinión sobre dinámicas efectivas y patrones ineficaces, comentarios sobre su impacto en el aprendizaje, sugerencias sobre cómo mejorar la coordinación entre compañeros o cualquier otra reflexión referente a este método de trabajo. La mayor parte de los participantes se muestra satisfecha con el desarrollo del proyecto hasta el momento, aunque subrayan la dificultad añadida que supone la imposibilidad de encontrarse físicamente para tratar ciertos aspectos del trabajo de forma más directa.

Al igual que en la anterior, en esta reunión se sigue buscando profundizar en la vertiente social de la iniciativa. En este caso, la discusión gira en torno al potencial del traductor como agente de cambio. Así, se conversa con los alumnos sobre cómo ven la que será probablemente su 
futura profesión y sobre si esta experiencia ha modificado esa visión en algún sentido. También se habla sobre cómo se sienten a medida que avanza el proyecto. Resulta gratificante comprobar que esta acción docente les ha hecho pensar, por primera vez en algunos casos, en la traducción como una herramienta de ayuda y un nivelador de asimetrías, una correa de transmisión de mensajes que transcienden lo instrumental o lo lúdico para penetrar en terrenos solidarios y formativos. Del mismo modo, reconocen que ver las posibilidades que ofrece esta ramificación social de la traducción les hace sentirse más orgullosos de haber escogido este camino y admiten que ver cada vez más cerca la compleción del proyecto les aporta una gran satisfacción personal.

La tercera fase de la acción docente implica aumentar el nivel de colaboración entre los miembros de la clase, ya que requiere la interacción entre los tres equipos ${ }^{3}$. Una vez el profesor devuelve los trabajos nuevamente revisados, este estadio busca que cada equipo pula los detalles de sus traducciones e interactúe con los otros dos para subrayar las principales diferencias entre las tres versiones así como aquellas dudas que no puedan resolver y necesiten consensuar con «el cliente», en este caso, los miembros de la ONG.

Estas preguntas serán la parte final de la presentación oral de los alumnos. Para facilitar su respuesta y dar tiempo a que los miembros de Manos Unidas escojan la solución que más les conviene, docente y discentes trabajan en una tabla que recoge las dudas de los estudiantes, así como las distintas alternativas planteadas por cada grupo cuando esta duda se base en la imposibilidad de decidir entre dos o más opciones. Los miembros de la ONG reciben esta tabla al final de la presentación y se la hacen llegar al profesor una vez completa. Al margen de estas cuestiones, la exposición de los estudiantes incluye una descripción de los pasos que han seguido para realizar la traducción, los principales problemas con los que se han encontrado, su opinión sobre la necesidad, utilidad y recorrido del proyecto, así como sus reflexiones finales en torno a esta experiencia.

La fase final del proceso consiste en el análisis e implementación de las respuestas de los miembros de Manos Unidas a las dudas planteadas por los estudiantes. Una vez recibidas, los alumnos se centran en dar forma a un último y único TM ajustado a las observaciones propuestas por los trabajadores de la ONG. Esto debería poner, en principio, punto y final al proyecto. Sin embargo, vista la acogida de todas las partes involucradas, hemos tratado de alargar el camino.

Pese a que ya no forme parte de la acción docente aquí descrita, se busca que el trabajo realizado tenga una proyección y continuidad para los participantes que así lo deseen en el futuro a corto plazo. De este modo, al cierre de este artículo, la Universidad de Alcalá y Manos Unidas negociaban un acuerdo para que puedan ser los propios estudiantes los que ejerzan como guías de la exposición, completando así un círculo en el que el alumnado opera como traductor de los materiales primero y como la persona encargada de difundir su contenido después.

\subsection{Recogida de datos}

Una vez finalizado el proyecto, se lanzaron dos encuestas de respuesta anónima creadas a través de la herramienta Google Forms, una entre los alumnos (encuesta 1 en adelante) y otra entre los miembros de la ONG participantes (encuesta 2). La primera pretende medir la utilidad que los estudiantes han concedido a la tarea con relación a sus estudios, pero también cómo esta ha impactado sobre su conciencia cívica, su motivación y su implicación en actividades de marcado

\footnotetext{
${ }^{3}$ Para la compleción de esta última fase, se sugiere la elección de un par de portavoces por equipo que canalicen las conversaciones entre los distintos grupos, pero también la interacción con el profesor y la presentación ante los miembros de la ONG.
} 
carácter social. Del mismo modo, ambos sondeos buscan calibrar la percepción sobre la continuidad de iniciativas de este tipo en próximos cursos, el potencial de la traducción como plataforma para propuestas de ApS, y el papel que los encuestados consideran que esta metodología debe ocupar en la educación superior.

Para ello, se formulan varias afirmaciones (nueve en el cuestionario dirigido a los estudiantes, tres en el dirigido a los miembros de la ONG) con las que los encuestados deben manifestar su conformidad o disconformidad a partir de una escala Likert de 1 a 5. Para evitar posibles sesgos, el punto 3 de la escala representa neutralidad ante la afirmación, por lo que la encuesta contempla un mismo número de reacciones positivas y negativas. A esto se suma un apartado final en el que los participantes pueden añadir, si lo desean, cualquier comentario u opinión adicional que no hayan reflejado con anterioridad. Asimismo, la encuesta 2 presenta dos preguntas de respuesta afirmativa o negativa que puede explicarse si se considerase oportuno. Todas estas decisiones buscan crear un cuestionario ágil y claro que no lleve más de cinco o diez minutos responder y que permita, por tanto, abordar cada punto con la atención necesaria.

Antes de distribuir las encuestas, se realizó una fase de pilotaje en la que colaboraron dos profesores de la Universidad de Alcalá, dos profesores externos y tres alumnos que habían participado en el proyecto. Los cuatro primeros, con amplia experiencia en la elaboración y desarrollo de encuestas, revisaron ambos sondeos, mientras que a los segundos solo se les hizo entrega de la encuesta dirigida al alumnado.

Como resultado del pilotaje se obtuvieron varias sugerencias que dieron lugar a los siguientes cambios:

- Se modificó el título en ambas encuestas para aclarar su propósito y contenido.

- Las dos secciones en las que en un principio estaba dividida la encuesta dirigida a los miembros de Manos Unidas se unieron y se añadió una explicación intermedia.

- Se incluyó un descriptor inicial en la encuesta dirigida al personal de la ONG en el que se describe y define brevemente la metodología aprendizaje-servicio.

- Se modificaron dos verbos para expresar posibilidad y no obligación.

- Se numeraron todas las preguntas sin distinción entre secciones en ambos casos.

- Se reordenaron algunas preguntas en el cuestionario dirigido al alumnado para que la progresión de la encuesta resultase más natural.

Entre los estudiantes, se obtuvieron 21 respuestas de 23 posibles ${ }^{4}$. Por su parte, los cuatro miembros de Manos Unidas involucrados en el proyecto respondieron en su totalidad al cuestionario. La relación final de preguntas junto con los resultados obtenidos en cada punto puede consultarse en las dos tablas incluidas a continuación.

\footnotetext{
${ }^{4}$ Los tres alumnos que realizaron el pilotaje no participaron en la encuesta final.
} 
1. Este proyecto me ha ayudado a concienciarme sobre problemas y necesidades de mi entorno más inmediato.
2. Este proyecto me ha animado a involucrarme en la solución de esos mismos problemas.

3. Participar en este proyecto ha enriquecido mis valores personales y aumentado $\mathrm{mi}$ responsabilidad cívica.

4. Este proyecto me ha permitido poner en práctica gran parte de los conocimientos obtenidos a lo largo del grado.

5. Este proyecto me ha ayudado a ver cómo los conocimientos obtenidos a lo largo del grado pueden tener una aplicación social y de apoyo a la comunidad.

6. Iniciativas de este tipo resultan motivadoras para el alumnado.

7. Iniciativas de este tipo ayudan a poner de relieve el papel social de la traducción.

8. Iniciativas de este tipo deberían tener un mayor espacio en el programa de las distintas asignaturas del grado.

9. Las asignaturas de traducción son un buen contexto en el que desarrollar iniciativas de este tipo.
Respuestas y porcentajes

Muy en desacuerdo: $4.8 \%$

Bastante en desacuerdo: $4.8 \%$

Neutral: $52.4 \%$

Bastante de acuerdo: $33.3 \%$

Muy de acuerdo: $4.8 \%$

Muy en desacuerdo: $4.8 \%$

Bastante en desacuerdo: $4.8 \%$

Neutral: 23.8\%

Bastante de acuerdo: $66.7 \%$

Muy en desacuerdo: $4.8 \%$

Neutral: $47.6 \%$

Bastante de acuerdo: $38.1 \%$

Muy de acuerdo: $9.5 \%$

Neutral: $9.5 \%$

Bastante de acuerdo: $28.6 \%$

Muy de acuerdo:61.9\%

Bastante en desacuerdo: $4.8 \%$

Neutral: $4.8 \%$

Bastante de acuerdo: $14.3 \%$

Muy de acuerdo: $76.2 \%$

Muy en desacuerdo: $4.8 \%$

Neutral: $4.8 \%$

Bastante de acuerdo: $9.5 \%$

Muy de acuerdo: $81 \%$

Bastante en desacuerdo: $4.8 \%$

Neutral: $4.8 \%$

Bastante de acuerdo: $9.5 \%$

Muy de acuerdo: $81 \%$

Bastante en desacuerdo: $4.8 \%$

Neutral: $4.8 \%$

Bastante de acuerdo: $9.5 \%$

Muy de acuerdo: $81 \%$

Neutral: $9.5 \%$

Bastante de acuerdo: $4.8 \%$

Muy de acuerdo: $85.7 \%$

Tabla 1. Encuesta realizada a los estudiantes 
Preguntas

1. Tras esta experiencia, ¿participarías en

proyectos similares en años venideros?

2. ¿Crees que la universidad debería impulsar más proyectos de este tipo?

\begin{tabular}{l|l}
\cline { 2 - 2 } & No \\
\hline \multirow{2}{*}{$\begin{array}{l}\text { 3. Los proyectos de aprendizaje-servicio } \\
\text { deberían ser parte esencial de la enseñanza }\end{array}$} & Muy en desacuerdo: \\
\cline { 2 - 2 } superior. & Neutral: \\
\cline { 2 - 2 } & Bastante de acuerdo: \\
\cline { 2 - 2 } & Muy de acuerdo:100\% \\
\hline \multirow{2}{*}{$\begin{array}{l}\text { 4. Los proyectos de aprendizaje-servicio } \\
\text { deberían ser parte esencial de la enseñanza de }\end{array}$} & Neutral: \\
\cline { 2 - 2 } la traducción en la universidad. & Bastante de acuerdo: \\
\cline { 2 - 2 } & Muy de acuerdo: $100 \%$ \\
\hline \multirow{2}{*}{$\begin{array}{l}\text { 5. La traducción es un campo idóneo para } \\
\text { explotar las posibilidades del aprendizaje- }\end{array}$} & Bastante en desacuerdo: \\
\cline { 2 - 2 } servicio. & Neutral: \\
\cline { 2 - 2 } & Bastante de acuerdo:25\% \\
\cline { 2 - 2 } & Muy de acuerdo:75\%
\end{tabular}

Respuestas

Sí: $100 \%$

No

Sí: $100 \%$

Tabla 2. Encuesta realizada a los miembros de Manos Unidas

\subsection{Discusión de resultados}

Las respuestas obtenidas aportan muchas certezas, si bien es cierto que dejan en el aire algunas cuestiones. En cuanto a las primeras, conviene subrayar distintos puntos. Por un lado, resulta destacable el abrumador porcentaje de encuestados que ve en la traducción y el ApS un binomio con enorme utilidad y posibilidades. Tanto los alumnos como los miembros de Manos Unidas coinciden en catalogar esta disciplina como idónea para el desarrollo de proyectos de aprendizaje-servicio. En el lado de los estudiantes, este dato entronca con el porcentaje similar de respuestas que lo consideran una buena o muy buena forma de consolidar los contenidos aprendidos a lo largo del grado, lo que demuestra que experiencias como esta pueden trascender una asignatura concreta e incardinarse en múltiples materias de un plan de estudios vertebrado por la traducción. Los dos puntos anteriores, unidos al componente motivador que más del $90 \%$ de los participantes ve en esta metodología, ayudan probablemente a explicar el deseo mayoritario de los discentes de que el ApS cobre un papel más central en sus estudios universitarios: 19 de los 21 participantes se muestran de acuerdo o muy de acuerdo con la posibilidad de que iniciativas de este tipo tengan mayor espacio en las distintas asignaturas ofertadas en el grado y recalcan su utilidad a la hora de poner de relieve el papel social que puede jugar la traducción.

Tanto este último dato como los resultados cosechados en la pregunta 5 son, en mi opinión, de suma relevancia, ya que, de algún modo, arrojan luz sobre las inconcluyentes cifras relacionadas con el fomento de la conciencia cívica del estudiantado. $Y$ es que, si bien los participantes no dan una respuesta contundente en lo que respecta a su efectividad para reforzar valores y actitudes asociados al bien común, sí que coinciden mayoritariamente en señalar que esta iniciativa les ha ayudado a ver la traducción y los conocimientos adquiridos como palancas de cambio con un importante valor añadido para la sociedad a distintos niveles. De este modo, las opiniones recogidas en la encuesta vienen a confirmar lo comentado en uno de los talleres 
anteriores y demuestran el éxito de la iniciativa a la hora de coadyuvar a construir un grupo de traductores más concienciados de lo imprescindible de su futura profesión, abiertos a explorar su vertiente más social y motivados para emplear lo aprendido en el grado en empresas solidarias que puedan cambiar sus entornos inmediatos $y$, quizás, también otros que no conocen.

Retomando la relación entre el ApS y los estudios universitarios, comprobamos que las opiniones de los estudiantes enlazan directamente con el parecer de los miembros de la ONG implicados en el proyecto. En su caso, todos consideran que esta metodología debería jugar un mayor papel en la Universidad española en general. Del mismo modo, la totalidad de los encuestados traslada esta afirmación a la enseñanza de la traducción, un campo que, como se señalaba anteriormente, consideran extremadamente propicio para el desarrollo del ApS. Estos resultados se concretan en el consenso en torno a futuras colaboraciones, quizás la más importante de las preguntas teniendo en cuenta la necesaria continuidad y expansión del ApS que defiende este artículo. En este caso, vuelve a imponerse el consenso: todos los miembros de la ONG encuestados se muestran abiertos a ellas y, de hecho, ya se han empezado a preparar futuros proyectos conjuntos.

Sin embargo, como se apuntaba al inicio de este apartado, no todas las preguntas han tenido una respuesta igualmente positiva y uniforme. Al margen de algún punto ya comentado en párrafos anteriores, en la zona templada del espectro encontramos los datos relativos al proyecto como herramienta de concienciación sobre los problemas del entorno, con un amplio número de alumnos mostrando neutralidad ante la afirmación en cuestión. Sin embargo, el proyecto sí ha aumentado su deseo de involucrarse en otras empresas de este tipo, lo cual puede llevarnos a colegir dos cosas. La primera, que la temática (y problemática) sobre la que gira la exposición de Súper Pepo les era relativamente familiar y no les ha descubierto ninguna necesidad nueva en su entorno. Esta suposición se ve confirmada, siquiera parcialmente, por opiniones como la siguiente, incluidas en el apartado final de comentarios de la encuesta: "La neutralidad de las primeras preguntas se debe a que considero que ya estaba concienciada e implicada (aunque sea desde mi humilde posición), entonces no he cambiado mi forma de ver las cosas, por así decirlo». La segunda conclusión que se puede sugerir a partir de estos datos es que, a pesar de una sensibilización general de partida, la propuesta sí que ha servido para propulsar sus ganas de contribuir en mayor medida e implicarse en labores solidarias de carácter similar.

\section{Conclusiones}

El propósito principal de este artículo era demostrar, a través de una experiencia didáctica real, la utilidad de las asignaturas de traducción como vehículo para introducir en los estudios universitarios de Grado algunos de los ejes centrales del aprendizaje-servicio (fomentar la responsabilidad cívica, crear sinergias entre teoría y práctica, potenciar la motivación de los estudiantes, orientar la educación a la mejora de la sociedad, favorecer la reflexión sobre lo aprendido). Los resultados obtenidos a lo largo de este estudio apoyan con considerable solidez esta realidad desde dos frentes: el del alumnado y el de la ONG involucrada en el proyecto. Ambos grupos consideran de forma casi unánime que este tipo de iniciativas deberían ser parte integrante de los programas de educación superior. Del mismo modo, ambos colectivos piensan que la traducción es un terreno fértil para el desarrollo de este tipo de iniciativas y coinciden en querer darles continuidad y un mayor espacio en los programas de asignaturas del área. Los participantes lanzan así un guante que los profesores deberíamos tratar de recoger, sobre todo teniendo en cuenta la más que positiva respuesta del alumnado tanto en el plano motivacional como en el académico. En este sentido, los datos recabados pueden servir como acicate para el 
impulso de nuevas propuestas orientadas a explotar todas las posibilidades que el binomio traducción-ApS ofrece.

Si bien es cierto que se trata de una muestra reducida y de una experiencia muy concreta, estas limitaciones, inherentes a casi toda acción docente, deberían verse no como una debilidad, sino como una invitación a la mejora y a la ampliación de horizontes. La sociedad en la que vivimos nos ofrece a diario muchas oportunidades de ayudar a los demás, y un gran número de ellas pasa necesariamente por facilitar o posibilitar la comunicación, a menudo interlingüística, entre las partes. Es ahí donde la traducción, en sus múltiples ramas y especialidades, juega y debe seguir jugando un papel fundamental al que la educación superior debe dar respaldo, ya sea aprovechando las numerosas posibilidades de colaboración con las ONG o explorando otros canales. La presente iniciativa, por tanto, empequeñece ante el vasto abanico de oportunidades que los estudios de traducción ofrecen al aprendizaje-servicio. Consciente de ello, estas páginas esperan haber sido, al menos, una piedra más en el camino hacia una Universidad y unos estudiantes y egresados más sensibilizados y abiertos a conocer e intervenir en las problemáticas que les rodean.

\section{Referencias bibliográficas}

Arrataia-Figueroa, A. (2008). Ética, solidaridad y "aprendizaje servicio" en la educación superior. Acta Bioethica, 14(1), 61-67.

Bell, R.T. (1991). Translation and translating. Londres: Longman.

Bugel, T. (2013). Translation as a multilingual and multicultural mirror framed by servicelearning. Hispania, 96 (2), 369-382.

Corsellis, A, Valero-Garcés, C.V. y Cobas-Álvarez, R. (2010). Traducción e interpretación en los servicios públicos: primeros pasos. Granada: Comares.

Council of Europe (2018). Common European framework of reference for languages: Learning, teaching, assessment. Companion volume with new descriptors. Obtenido de https://rm.coe.int/cefr-companion-volume-with-new-descriptors-2018/1680787989

Faszer-McMahon, D. (2013). Social networking, microlending, and translation in the Spanish service-learning classroom. Hispania, 96 (2), 252-263.

Francisco-Amat, A. y Moliner-Miravet, L. (2010) El Aprendizaje Servicio en la Universidad: una estrategia en la formación de ciudadanía crítica. Revista Electrónica Interuniversitaria de Formación del Profesorado, 13(4), 69-77. Obtenido de https://www.redalyc.org/pdf/2170/217015570006.pdf

Gil-Sanromán, I. (2019). Implantación del programa aprendizaje-servicio en traducción especializada. Universidad Pontificia Comillas.

Hurtado-Albir, A. (1996). La enseñanza de la traducción. Castellón: Universitat Jaume I.

Kelly, D. (2002). Un modelo de competencia traductora: bases para el diseño curricular. Puentes: Hacia nuevas investigaciones en la mediación intercultural, 1, 9-20.

Madrid, A. (2008). El proyecto derecho al Derecho: un planteamiento de actuación y reflexión comunitario. En Martínez, M. (ed.) Aprendizaje servicio y responsabilidad social de las universidades, pp.93-112. Barcelona: Octaedro.

Maldonado-Pérez, M. (2007). El trabajo colaborativo en el aula universitaria. Laurus: Revista de Educación, 13, 263-278. 
Maldonado-Pérez, M., y Sánchez, T. (2012). Trabajo colaborativo en el aula: experiencias desde la formación docente. Revista EDUCARE, 16(2), 93-118.

Mareschal, G. (2005). L'enseignement de la traduction au Canada. Meta, 50(1), 250-262.

Martín, X. y Puig, J.M. (2018). Aprendizaje-servicio: conceptualización y elementos básicos. En Serrano, L. R., y A. E. Roig (eds.). Aprendizaje-Servicio (ApS): claves para su desarrollo en la Universidad. Barcelona: Octaedro.

Martínez, M. (2018). Prólogo. En Serrano, L. R. y A. E. Roig (eds.). Aprendizaje-Servicio (ApS): claves para su desarrollo en la Universidad. Barcelona: Octaedro.

Martínez-Odría, A. (2005). Service-Learning o Aprendizaje-Servicio: una propuesta de incorporación curricular del voluntariado. Universidad de Navarra.

Neubert, A. (2000). Competence in language, in languages and in translation developing. En Adab, B. y C. Schaffner (eds.). Translation competence, pp. 3-18. Ámsterdam: John Benjamins.

Pérez-Pueyo, A., Hortigüela-Alcalá, D., González-Calvo, G. y Fernández-Río, J. (2019). Muévete conmigo, un proyecto de aprendizaje servicio en el contexto de la educación física, la actividad física y el deporte. Publicaciones, 49(4), 183-198.

Revilla-Blanco, M. (2015). Las ONG en España. En Alonso Benito, L.E. y M. Revilla Blanco. España 2015: situación social, pp. 1160-1173. Centro de Investigaciones Sociológicas. Obtenido de https://eprints.ucm.es/44319/1/Revilla\%200NG\%20en\%20Espa\%C3\%B1a.pdf

Rodríguez-Gallego, M.R. (2014). El Aprendizaje Servicio como estrategia metodológica en la universidad. Revista Complutense de Educación, 25 (1), 95-113.

Sales, D. (2005). Panorama de la mediación intercultural y la traducción e interpretación en los servicios públicos. Translation Journal, 9 (1). Obtenido de http://translationjournal.net/journal/31mediacion.htm

Tamayo, A. (2016). Subtitulación para personas sordas: metodología de aprendizaje-servicio en el aula de traducción audiovisual. Íkala, revista de lenguaje y cultura, 21(3), 327-342.

Valero-Garcés, C y Cata, L. (2006). Acción y voluntariado: las ONG y los servicios de traducción e interpretación. Revista española de lingüística aplicada, (1), 49-60.

Vázquez-Navarrete, M. L., Terraza-Núñez, R., Vargas-Lorenzo, I. y Lizana-Alcazo, T. (2009). Necesidades de los profesionales de salud en la atención a la población inmigrante. Gaceta Sanitaria, 23(5), 396-402. 